\title{
HEALTH POLICY \\ Health Care Providers' Experiences with Implementing Medical Aid-in-Dying in Vermont: a Qualitative Study
}

\author{
Mara Buchbinder, $P h D^{7}$, Elizabeth R. Brassfield, $M A^{2}$, and Manisha Mishra, $B A^{3,4}$ \\ 'Department of Social Medicine, Center for Bioethics, University of North Carolina at Chapel Hill, Chapel Hill, NC, USA; ${ }^{2}$ Department of Philosophy \\ and School of Medicine, University of North Carolina at Chapel Hill, Chapel Hill, NC, USA; ${ }^{3}$ University of North Carolina at Chapel Hill, Chapel Hill, NC, \\ USA; ${ }^{4}$ Present Address: Center for Medicine, Health, and Society at Vanderbilt University, Nashville, TN, USA.
}

BACKGROUND: The evolving legal landscape for medical aid-in-dying (AID) in the USA raises clinical and public health challenges and concerns regarding how health care providers will accommodate AID while expanding access to high-quality end-of-life care.

OBJECTIVE: To describe Vermont health care providers' experiences practicing under the "Patient Choice and Control at End of Life" Act.

DESIGN: Qualitative semi-structured interviews analyzed using grounded theory.

PARTICIPANTS: The larger study included 144 health care providers, terminally ill patients, caregivers, policy stakeholders, and other Vermont residents working in 10 out of Vermont's 14 counties. This article reports on a subset of 37 providers who had clinical experience with the law.

MAIN MEASURES: Themes from interviews.

KEY RESULTS: Physicians were roughly split between hospital and community-based practices. Most were women (68\%) and the largest subgroup specialized in internal or family medicine (53\%). Most of the nurses and social workers were women (89\%) and most worked for hospice and home health agencies (61\%). We identified five domains in which participants engaged with AID: (1) clinical communication and counseling; (2) the Act 39 protocol; (3) prescribing medication; (4) planning for death; and (5) professional education. How providers experienced these five domains of clinical practice depended on their practice setting and the supportive resources available.

CONCLUSION: Health care providers' participation in AID involves clinical tasks outside of responding to patients' requests and writing prescriptions. Research to identify best practices should focus on all domains of clinical practice in order to best prepare providers.

KEY WORDS: medical aid-in-dying; end-of-life care; ethics; qualitative research; health policy.

J Gen Intern Med 34(4):636-41

DOI: $10.1007 / \mathrm{s} 11606-018-4811-1$

(c) Society of General Internal Medicine 2019

Electronic supplementary material The online version of this article (https://doi.org/10.1007/s11606-018-4811-1) contains supplementary material, which is available to authorized users.

Received May 3, 2018

Revised October 22, 2018

Accepted December 6, 2018

Published online January 25, 2019

\section{INTRODUCTION}

The legal landscape for medical aid-in-dying (AID) in the USA has shifted dramatically over the past 5 years. Vermont (2013), California (2015), Colorado (2016), the District of Columbia (2016), and Hawaii (2018) have joined Oregon (1997), Washington (2008), and Montana (2009) in permitting physicians to prescribe a lethal dose of medication to a terminally ill adult patient, provided that certain conditions are met. With the extension of this option to over 39 million people in California, almost $20 \%$ of the US population now lives in a jurisdiction in which AID is permitted.

This evolving legal landscape raises clinical and public health challenges and concerns regarding how health care providers, institutions, and medical systems will handle requests for AID while expanding access to high-quality end-oflife care. ${ }^{1}$ To address these challenges, both opponents and proponents have issued calls for more robust data collection. Additional data can address concerns about abuses and errors in implementation, ${ }^{2,3}$ as well as the concerns of those who want to ensure that the practice works as intended to serve patients' end-of-life goals. ${ }^{1,}{ }^{4}$ Prior studies have focused on physicians' willingness to prescribe and how they respond to requests for hastened death. ${ }^{5-10}$ Several reports have also provided data about the utilization of AID in clinical contexts $^{11-13}$ and the development of institutional policies. ${ }^{12,14}$ ${ }^{17}$ However, more information is needed about how individual health care providers have worked within the constraints of institutional policies and navigated practical challenges relating to interpreting and implementing these new laws. Such challenges may include gaps in relevant clinical knowledge or details of the law, concerns about privacy or the potential for legal or social ramifications, and other process-based issues. ${ }^{18}$

This article describes Vermont health care providers' experiences practicing under the "Patient Choice and Control at End of Life Act" (Act 39), ${ }^{19}$ which passed in May 2013. Paperwork for 52 AID cases was submitted between May 2013 and July 2017 under the Act 39 protocol; 83\% have involved cancer diagnoses and $14 \%$ ALS diagnoses. ${ }^{20} \mathrm{We}$ report on findings from The Vermont Study on Aid-in-Dying (Vermont SAID), which documented how physicians, nurses, and social workers engaged with AID in a variety of ways that extend beyond responding to patients' requests or deciding 
whether or not to prescribe. We identify and describe five domains in which health care providers participate in the implementation of AID. We highlight, in particular, that even if a physician ultimately declines to prescribe, she may participate in other domains of care, and that nurses and social workers play a critical role in these emerging clinical duties.

\section{METHODS}

\section{Research Design, Setting, and Overview}

Vermont SAID was a qualitative, descriptive study. ${ }^{21}$ The goal of the study was to characterize the implementation and social impact of Act 39 in the period following legalization. Vermont was selected for the case study because it had most recently legalized AID when the study began, providing a valuable opportunity to observe responses to the law as they unfolded longitudinally. The small size and geographic proximity of key institutions also made it possible for the principal investigator (MB), who carried out the fieldwork independently, to document the law's effects in lay, medical, and legislative settings across the state. The study was approved by the Institutional Review Board at the University of North Carolina at Chapel Hill.

Data from Vermont SAID include (1) 144 semi-structured in-depth interviews with physicians $(n=29)$; nurses, chaplains, and social workers $(n=22)$; terminally ill patients $(n=$ $9)$; caregivers $(n=34)$; activists, legislators, and other policy stakeholders $(n=37)$; and additional Vermont residents without a direct stake in Act $39(n=13)$, and (2) ethnographic observations in community-based advocacy and educational events and professional medical conferences. For this analysis, we focus on interviews conducted with a subset of health care providers who had clinical experience with Act 39.

\section{Data Collection}

Providers were recruited via an opportunistic and nonprobabilistic sampling approach, resulting in a convenience sample. Potential participants were identified using online searches to generate a list of physicians in departments of oncology, neurology, and palliative care - specialties where the largest numbers of AID patients are treated - across state hospitals. These individuals were then invited to participate via email, telephone, or letter. Hospice agency representatives were contacted similarly and asked to convey information about the study to relevant staff. A study announcement was also circulated on the Vermont Medical Society listserv. A small number of participants were recruited through face-toface meetings at medical conferences and advocacy events. Additional recruitment relied on a snowball sampling approach, particularly effective for accessing expert populations. ${ }^{22}$ Study participants were asked to share information about Vermont SAID with other healthcare providers in their personal and professional networks.

A semi-structured interview guide previously used to study clinical adaptations to a new state abortion law was modified for use with Vermont health care providers. ${ }^{23}$ The interview guide included the following domains: (1) professional background, (2) ideas about a "good death," (3) views on the provider's role in end-of-life care, (4) attitudes toward AID, (5) interactions with patients and families about AID, and (6) views on the consequences of Act 39. Interview questions were broad and open-ended to capture a breadth of responses. (See online Appendix.) This article reports on interview responses from domains (5) and (6), but other domains were included to provide contextual information relevant to the project's broader aims.

Data were collected over 22 weeks between July 2015 and July 2017. MB, a medical anthropologist with expertise in qualitative methods, generally conducted interviews in person, in a location of the participant's choosing, such as the participant's home, medical office, or a local café. One interview was conducted by telephone when a suitable in-person meeting time could not be arranged. At the outset of the interview, participants provided written informed consent. Interviews were recorded using digital audio-recorders but participants could opt out of recording and still participate. Five participants (including one in this sub-study) declined to be recorded. Recordings were supplemented by written field notes that included contextual details and key points. Interviews lasted between 39 and 106 min. Participants were not compensated for their time.

\section{Data Analysis}

Data analysis was completed between October 2017 and February 2018. After interview recordings were transcribed verbatim and deidentified, transcripts were analyzed using an inductive, iterative approach guided by the tenets of grounded theory. ${ }^{24}$ First, $\mathrm{MB}$ and two research assistants (EB and MM) read transcripts to identify emergent themes. Themes were broadly defined to capture depth and variation across participants' experiences and then organized into a structured coding dictionary that included a definition for each of the 44 codes. Codes were assigned to excerpts of interview text that matched the code definition using NVivo 11 Software (QSR International, Melbourne, Australia). Transcripts and field notes of unrecorded interviews were coded by two coders blinded to the other's work, and discrepancies were discussed and resolved so that understanding of concepts and codes remained in agreement. After coding was complete, several themes most frequently discussed by health care providers were identified and coding reports from these themes were further examined to identify patterns across the larger data set.

For this analysis, providers were excluded if they did not have direct experience with Act 39, which we defined as having provided clinical care for a patient who initiated the Act 39 protocol or having assisted a patient with navigating access to Act 39 . We made these determinations by closely reviewing coded excerpts from the "provider role" code (i.e., general statements about a provider's roles, duties, and obligations with respect to Act 39). Nurses and social workers as 
well as physicians were included because both groups contributed important information about the role of health care providers in implementing Act 39. We determined that analyzing data from both groups together would result in a more robust analysis than analyzing either subgroup separately, particularly because participants spoke about the roles of their clinical colleagues.

\section{RESULTS}

This article reports on interviews with a subset of 37 providers in the Vermont SAID sample who had clinical experience with Act 39. Physicians were roughly split between hospital and community-based practices, with most $(53 \%)$ specializing in internal or family medicine (see Table 1 ). Their mean age was 51.3. At the time of interview, of the 19 physicians, 12 had participated in Act 39 as prescribing physicians; the remainder had initiated but not completed the Act 39 protocol $(n=3)$, participated as a second physician to confirm the patient's diagnosis, prognosis, and decisional capacity $(n=3)$, or counseled patients $(n=1)$. The mean age of nurses and social workers was 52.5, with most working for hospice and home health agencies $(61 \%)$. While all professionals in this group engaged in clinical care for patients pursuing Act 39, specialty clinic nurse practitioners were more likely to assist with navigating access to AID. Participating health care professionals worked in ten of Vermont's 14 counties.

We identified five domains in which participants engaged with AID after legalization: clinical communication and counseling, the Act 39 protocol, prescribing medication,

Table 1 Participant Demographics

\begin{tabular}{|c|c|}
\hline Characteristics & $N(\%)$ \\
\hline \multicolumn{2}{|l|}{ Physicians } \\
\hline \multicolumn{2}{|l|}{ Specialty } \\
\hline Internal/family medicine & $10(53 \%)^{*}$ \\
\hline Hospice/palliative care & $4(21 \%)$ \\
\hline Neurology & $3(16 \%)$ \\
\hline Oncology & $2(11 \%)$ \\
\hline \multicolumn{2}{|l|}{ Practice type } \\
\hline Hospital-based & $10(53 \%)$ \\
\hline Community & $9(47 \%)$ \\
\hline \multicolumn{2}{|l|}{ Sex } \\
\hline Female & $13(68 \%)$ \\
\hline \multicolumn{2}{|l|}{ Years in practice } \\
\hline$<10$ & $4(21 \%)$ \\
\hline $11-20$ & $6(32 \%)$ \\
\hline $21-30$ & $7(37 \%)$ \\
\hline$>30$ & $2(11 \%)$ \\
\hline \multicolumn{2}{|l|}{ Nurses and social workers } \\
\hline \multicolumn{2}{|l|}{ Specialty } \\
\hline Hospice/home health nurse & $9(50 \%)$ \\
\hline Hospital-based specialty clinic nurse practitioner & $5(28 \%)$ \\
\hline Inpatient palliative care nurse & $2(11 \%)$ \\
\hline Hospice social worker & $2(11 \%)$ \\
\hline \multicolumn{2}{|l|}{ Sex } \\
\hline Female & $16(89 \%)$ \\
\hline Male & $2(11 \%)$ \\
\hline
\end{tabular}

*Percentages may total more than 100 due to rounding planning for death, and professional education. We elaborate on these domains below and provide examples and illustrative quotations from the interviews in Table 2.

\section{Clinical Communication}

Participants emphasized that reassuring patients that they would be there for them and exploring their reasons for requesting AID were important first steps. Providers were particularly attuned to probing for concerns about finances or being a burden on others due to terminal illness, and often framed Act 39 as a last-resort option. Before seriously pursuing Act 39, providers sought to address untreated symptoms, such as pain and depression, and discussed alternative options for end-of-life care, including hospice, palliative care, and voluntary stopping of eating and drinking. One nurse noted that after a patient brings up Act 39, "I feel a little bit more free [sic] to discuss with people the ways that they can maybe hasten or allow the dying process to occur without involving medical assistance" (0123). Only then would they educate patients about the law, including who qualifies and how it works. Many providers noted that discussing AID could open up conversations about other end-of-life concerns. As one physician puts it, "There are more people who can say, I think, 'Can I have that death pill?' than who can say, 'What can you do for me while I'm dying that I'm not gonna suffer?'” (0111). Several physicians noted that these issues were difficult to address within a 15-30-min office visit.

\section{Act 39 Protocol}

All providers emphasized that understanding the law is timeconsuming and that it is difficult to grasp the details before beginning the process (see Box 1). Providers noted that Act 39 gives physicians discretion to determine who counts as a state resident, which sometimes put physicians in an uncomfortable position because determining residency lay outside the scope of their professional judgment. Some physicians felt uneasy with the law's competency requirement because there was no mechanism to ensure that the patient retained capacity if she did not utilize the prescription immediately. Others noted that prognostic uncertainty made it difficult to confirm the eligibility criteria, which include expected death within 6 months. Nurses and social workers contributed to other aspects of the Act 39 protocol, such as completing paperwork, finding witnesses, and helping patients to find a second physician. Physicians working in academic specialty clinics found this support helpful, but such support was less available to primary care physicians, particularly those working in community practices. Physicians working in hospital-based practices sometimes felt compelled to consult their hospital's legal or ethics services before agreeing to prescribe. Many prescribing physicians made house calls at some point to relieve patients of having to make additional office visits for completing the Act 39 protocol, which includes two oral, in-person requests spaced 15 days apart. 
Table 2 Domains of clinical practice: sub-themes and illustrative quotations

Clinical Communication and
Understand the request
Address untreated
symptoms
Discuss alternatives
Explain the law
Talk with family members
Frame as last-resort option

Act 39 Protocol

Verify eligibility

Complete paperwork

Identify and coordinate

with pharmacy

Identify second physician

Consult legal and/or ethics service

\section{Prescribing}

Select medication and

dosage

Consult other physicians

Determine cost

\section{Planning for Death}

Determine who will be present for the death

Determine who will pronounce the death

Make contingency plans

\section{Counseling} community who was on board with it,
"If someone says, 'I want you to help me die, I want Act 39,' the first question is, tell me more. And we deepen the conversation." (Physician, 0101)

"There is an obligation to do everything you can to try to keep them from feeling like they need to use that. You don't just give them the gun without the gun education, you know...You just loaded the gun for them and you need to make sure that they understand what they're doing, and that everything has been done to treat their distress." (Physician, 0110)

"His primary doctor...was unsure about his timeline, couldn't say for sure he had six months or less. But his neurologist and our hospice medical director who had been seeing him both said, 'Absolutely he has a prognosis of six months or less, and to come on to hospice services in the first place, you have to have that prognosis.' So, it was kind of interesting to see physicians butting heads." (Nurse, 0135) "We had to work with him and find, you know, another physician in the and that was not an easy task, took a lot of education, effort, support to other providers." (Nurse, 0131) "Turns out all he had to do was register to vote to say he was going to be, that he was legal resident. The law left open that established care and established residency, they left up to the discretion of the doctor. We didn't have any guidelines for it and we just didn’t know." (Nurse, 0119)

"The state website has the process. They don't have the how to. And so that's a little hard to do. I think in the end I contacted ( advocacy organization)) ... I ended up getting a hold of a physician out in, uh, ((name of city in another state)), I think. And he told me about the various protocols." (Physician, 0129)

"That's why we had a doctor and a social worker at the house at the same time, because we thought that this might happen, that the client would not be able to finish the cocktail." (Physician, 0138)

"In the last couple of days, she suddenly couldn't swallow it that quickly. ... I remember we talked about this, I'm like, 'What are you going to do, what's the plan if it doesn't work?' We spent a lot of time talking about that." (Physician, 0141) "Even though I'm not the prescriber, we talked a lot about what happens if it doesn't work. You know, what other medications do you have in the house and who would use them and who would be called. Even our triage office staff made sure everyone had our number. ... I think we all felt kind of an individual obligation to make sure

\section{Table 2. (continued)}

\begin{tabular}{ll}
\hline \hline & $\begin{array}{l}\text { that it went the way it was supposed to } \\
\text { and the best way to do that was to put } \\
\text { all of our heads together and try to } \\
\text { preplan as much as we could." } \\
\text { (Physician, 0145) }\end{array}$ \\
$\begin{array}{l}\text { Professional Education } \\
\text { Share experience with AID } \\
\text { informally or formally }\end{array}$ & $\begin{array}{l}\text { "I've offered to do talks at the CMEs } \\
\text { or different situations and [hospital } \\
\text { administrators] refused to let me talk } \\
\text { about Act 39 at all. I'm not talking } \\
\text { about trying to talk them into doing it. } \\
\text { I'm just trying to talk to them about if } \\
\text { they have a patient who comes in who } \\
\text { is interested in it, here is where you } \\
\text { can get information on it." (Physician, } \\
0134)\end{array}$ \\
\hline
\end{tabular}

\section{Prescribing}

Far from the one-time event of writing a script, prescribing was a multi-dimensional practice that involved figuring out what medication and dose to prescribe, determining cost and insurance coverage, and investigating alternative protocols if cost proved to be a barrier (see Box 2). Because few physicians had local colleagues with the relevant clinical experience, prescribing frequently involved consultation with out-of-state physicians. One physician noted that, although there had been other AID cases in her hospital, her patient was the first diagnosed with an obstructive gastrointestinal cancer and she had logistical questions about the patient's ability to swallow the medication that she discussed with an out-of-state physician. While the burdens of prescribing fell primarily to physicians, nurses and social workers, when available, occasionally helped to identify pharmacies and determine insurance coverage.

\section{Planning for Death}

Providers reported guiding patients about how the medication should be prepared (e.g., mixing with applesauce) and how fast it must be consumed. In many cases, providers spent substantial time planning for the day of the death, addressing

\section{Box 1 Case study: navigating the Act 39 protocol}

Dr. Copeland* was a hospital-based primary care physician who was approached by a new patient with ALS, Jim, who had moved to Vermont with the hope of accessing Act 39. Dr. Copeland felt uncertain about prescribing but understood Jim's reasons for pursuing AID and eventually agreed to participate. She had no experience with AID and the lack of infrastructure to support providers through the process meant that she had to spend significant time making phone calls to work out logistics. Dr. Copeland learned where to find the necessary forms for documentation, what type and dose of medication to prescribe, which pharmacy would fill the prescription, and who could assist, in what ways, with administering the drug. She also consulted with her legal department to determine who could be present for the death and what responsibility she would have for any complications that might arise. Because Jim had moved recently, Dr. Copeland also had to figure out whether he met the law's residency requirement. Jim asked Dr. Copeland to be present for his death, but she ultimately decided against attending. After this experience, Dr. Copeland and several colleagues held a lecture to educate their institution about her experience with Act 39 .

*All names are pseudonyms. 
Box 2 Case study: committing to non-abandonment

Dr. Rhodes*, a hospice and palliative care physician, was approached by Jacob, a patient in his late 60s diagnosed with late-stage cancer. Jacob was a veteran who had lived a solitary life after a brief marriage. He had no relationships with family and did not want to pursue any medical treatment because he did not want to become dependent on caregivers. Jacob had investigated various methods of suicide but determined that Act 39 would cause the least distress for others. Dr. Rhodes felt surprised by her ambivalence. She viewed prescribing as bearing a tremendous moral and emotional responsibility and she did not take the decision lightly. Yet she understood Jacob's choice and saw it as rational because it reflected the way he had lived his entire life. Moreover, she was committed to non-abandonment, and she was convinced that if she did not write the prescription, Jacob would end his life in a more gruesome manner. Dr. Rhodes consulted with experienced colleagues and a pharmacist about the protocol. She also contacted Jacob's hospice nurse to make sure that she felt comfortable caring for him. Jacob chose to be alone for his death, which required some coordination with his hospice nurse so that she could care for him afterward. Prior to Jacob's death, Dr. Rhodes visited him in his home to say goodbye.

*All names are pseudonyms.

questions about whether the patient could ingest the medication quickly enough for it to be effective and who would be on call to deal with unanticipated complications. Hospice nurses, in particular, reported some strains around dealing with adverse events: "We're now making sure we record who the prescriber is to make sure they're available at that moment because we don't want them to think hospice is gonna be the one, you know, at 2:00 in the morning ... they need to help us" (Nurse, 0132). Providers also considered whether they were personally willing to attend an assisted death if a patient requested that they do so, and if not, which other caregivers would be present to support the patient. Only six providers (two nurses and four physicians) (16.2\%) in the sample reported having attended a patient's death.

\section{Education}

Providers lamented the lack of formal education, training, and institutional support around Act 39, and reported seeking out information through informal professional networks and advocacy organizations, such as Compassion and Choices" "Doc2Doc" program. Several physicians expressed a commitment to educating colleagues about the process, both formally and informally, illustrating that the provider's role in AID does not necessarily end with a patient's death. One physician organized a presentation at her hospital to share her experience in navigating the protocol (see Box 1). Another physician scheduled a community dialog at his hospital for colleagues to share views about AID.

\section{Synthesis}

How providers experienced the five domains of AID clinical practice depended on their practice setting and the supportive resources available. The extent to which providers felt burdened by these clinical duties was a function of the extent to which Act 39 had been institutionalized in their practice settings. Local experience, the existence of institutional policies to guide the practice, and the availability of social and emotional support from colleagues varied widely. Physicians bore the primary burden of prescribing and educating colleagues. Consequently, a small number of physicians expressed safety concerns regarding possible backlash from opponents of AID, particularly given that many providers were based in small rural communities where "everybody knows where I live" (Physician, 0108). Concerns about how participating in AID might affect one's relationships with colleagues who opposed the practice were relayed more commonly. Nevertheless, three physicians also expressed tremendous pride in their work. As one physician shared, "At the beginning of this, the oncologist and I would talk about being known as the death doctors around here. And we said it jokingly, but as time goes on, I'm actually kind of proud of that, that I'm one of the people who will do this" (0133).

\section{DISCUSSION}

Although the clinical literature on AID often focuses on the challenge of responding to patients' requests, ${ }^{25-27}$ this study of the experiences of physicians, nurses, and social workers with implementing Act 39 in Vermont documented a wider range of ways in which experts and professional societies ought to prepare health care professionals to engage with the practice. This article presents five domains in which providers participate in the implementation of AID: clinical communication and counseling, Act 39 protocol, prescribing, planning for death, and professional education. One advantage of these conceptual domains for researchers and policymakers is that they highlight the ambivalence that many health care professionals feel, insofar as they may decide to engage in clinical tasks in one domain, yet not in others (e.g., prescribing). Therefore, these domains enable a more nuanced understanding of health care providers' participation in AID, challenging the notion that they are either starkly willing or unwilling to participate.

Strengths of this study include its diverse sample, which contained health care providers from various clinical specialties and practice environments, and its qualitative, descriptive approach to understanding the practice of AID. The study also has several limitations. Vermont is a small, rural state with limited racial and ethnic diversity, which may limit generalizability. Further research is necessary to determine how these domains of participation apply to other recently permissive jurisdictions. Vermont SAID also utilized a convenience sample that may not represent the experiences of all Vermont providers. Nevertheless, given the low utilization in the state (i.e., 52 cases between 2013 and 2017), ${ }^{20}$ we believe that our sample of 37 providers represents a generous cross section of Vermont providers who have participated in Act 39.

This study makes a valuable contribution to the growing literature on AID in the USA, which was primarily derived from Oregon and Washington. The emerging clinical picture suggests that context matters greatly, and that institutional and systemslevel variables affect how it is implemented and accessed. In California, participation has been managed at the institutional level, with individual health systems "opting in" to AID, ${ }^{11,16}$ 
whereas Vermont did not follow a coordinated institutional approach to implementation. While it is widely recognized that all US statutes are based on Oregon's Death with Dignity Act, it is vital to understand contextual variation in implementation.

Finally, previous qualitative studies of AID have not incorporated perspectives from nurses and social workers, despite their intimate involvement with patients. ${ }^{28-30}$ This study suggests that nurses and social workers play a critical, yet underrecognized role in supporting patients to navigate access and in alleviating some of the practical burden on physicians.

Participating in AID takes considerable time, much of which may be unremunerated. Providers desire more formalized education about clinical and logistical aspects of AID protocols. Many providers who participate report that doing so is emotionally stressful, despite expressing gratitude for the ability to support patients' end-of-life wishes. To the extent that bolstering responses to requests for AID may represent an important opportunity to improve end-of-life care for all patients, ${ }^{31}$ more research is needed to identify best practices.

Contributors: Lauren Brinkley-Rubinstein provided helpful comments on a draft of this manuscript.

Corresponding Author: Mara Buchbinder, PhD; Department of Social Medicine, Center for Bioethics University of North Carolina at Chapel Hill, Chapel Hill, NC, USA (e-mail: mara_buchbinder@med. unc.edu).

Funders This research was supported by a UNC Junior Faculty Development award, a Greenwall Faculty Scholars Award, and a research grant from the National Science Foundation (1630010).

Data Availability A subset of the data analyzed during the current study is available from the corresponding author on reasonable request, and through ICSPR (https://www.icpsr.umich.edu/ icpsrweb/ICPSR/) after February 1, 2019. Some interview transcripts will not be made publicly available because participants were permitted to opt out of data archival when providing informed consent.

\section{Compliance with Ethical Standards:}

Conflict of Interest: The authors declare that they do not have a conflict of interest.

Publisher's Note: Springer Nature remains neutral with regard to jurisdictional claims in published maps and institutional affiliations.

\section{REFERENCES}

1. Ganzini L, Back AL. The challenge of new legislation on physicianassisted death. JAMA 2016;176(4):427-428.

2. Hendin H, Foley K. Aid-in-dying in Oregon: a medical perspective. Mich Law Rev 2008;106:1613-1640.

3. Sulmasy LS, Mueller PS. Ethics and the legalization of physician-assisted suicide: An American College of Physicians Position paper. Ann Intern Med 2017; 167(8):576-578.

4. Quill TE, Arnold RM, Youngner SJ. Aid-in-dying: finding a path forward in a changing legal environment. Ann Intern Med 2017;167(8):597-598.

5. Back AL, Starks H, Hsu C, Gordon JR, Bharucha A, Pearlman RA. Clinician-patient interactions about requests for aid-in-dying. Arch Intern Med 2002;162(11):1257-1265.
6. Back, AL. Doctor-patient communication about aid-in-dying. In: PhysicianAssisted Dying: The Case for Palliative Care and Patient Choice. Quill TE and Battin MP, eds. Baltimore: Johns Hopkins University Press, 2004:102-117.

7. Dobscha SK, Heintz RT, Press N, Ganzini L. Oregon physicians' responses to requests for assisted suicide: a qualitative study. J Palliat Med 2004;7(3):451-461.

8. Kohlwes RJ, Koepsell TD, Rhodes LA, Pearlman RA. Physicians' responses to patients' requests for aid-in-dying. Arch Intern Med 2001;161(5):657-663.

9. Voorhees JR, Rietjens JAC, van der Heide A, Drickamer MA. Discussing physician-assisted dying: physicians' experiences in the United States and the Netherlands. The Gerontologist 2014;54(5):808-17.

10. Ganzini L, Nelson HD, Schmidt TA, Kraemer DF, Delorit MA, Lee MA Physicians' experiences with the Oregon Death with Dignity Act. N Engl J Med 2000;342(8):557-563.

11. Nguyen HO, Gelman EJ, Bush TA, Lee JS, Kanter MH. Characterizing Kaiser Permanente Southern California's experience with the California End of Life Option Act in the first year of implementation. JAMA Intern Med 2018;178(3):417-421.

12. Loggers ET, Starks H, Shannon-Dudley M, Back AL, Appelbaum FR, Stewart FM. Implementing a death with dignity program at a comprehensive cancer center. N Engl J Med. 2013;368(15):1417-24.

13. Wang LH, Elliott MA, Jung Henson L, Gerena-Maldonado E, Strom S, Downing S, et al. Death with dignity in Washington patients with amyotrophic lateral sclerosis. Neurology 2016;87(20):2117-2122.

14. Campbell CS, Black MA. Dignity, death, and dilemmas: a study of Washington hospices and physician-assisted death. J Pain Symptom Manage 2014;47(1):137-153.

15. Campbell CS, Cox JC. Hospice-assisted death? A study of Oregon hospices on death with dignity. Am J Hosp Palliat Med 2012;29(3):227-235.

16. Harman SM, Magnus D. Early experience with the California End of Life Option Act: balancing institutional participation and physician conscientious objection. JAMA Intern Med 2017;177(7):907-908.

17. Petrillo LA, Dzeng E, Harrison KL, Forbes L, Scribner B, Koenig BA. How California prepared for implementation of physician-assisted death: a primer. Am J Public Health 2017;107(6):883-888.

18. Cain, C Implementing Aid in Dying in California: Experiences from Other States Indicates the Need for Strong Implementation Guidance. 2016; Policy Brief: UCLA Center for Health Policy Research: (PB2016-4)1-8.

19. Vermont Act 39: An act relating to patient choice and control at end of life. 2013.

20. Vermont Department of Health Report to the Vermont Legislature Concerning Patient Choice at the End of Life. 2018 https://legislature. vermont.gov/assets/Legislative-Reports/2018-Patient-Choice-Legislative-Report-12-14-17.pdf. Accessed November 10, 2018.

21. Sandelowski, M. Whatever happened to qualitative description? Res Nurs Health. 2000;23(4):334-340.

22. Bernard HR. Research Methods in Anthropology: Qualitative and Quantitative Approaches. Lantham: AltaMira Press; 2011.

23. Mercier RJ, Buchbinder M, Bryant A, Britton L. The experiences and adaptations of abortion providers practicing under a new TRAP law: a qualitative study. Contraception 2015;91(6):507-512.

24. Corbin J, Strauss A. Basics of Qualitative Research: Techniques and Procedures for Developing Grounded Theory. Thousand Oaks: Sage Publications; 2007.

25. Quill TE, Back AL, Block SD. Responding to patients requesting physician-assisted death: physician involvement at the very end of life. JAMA 2016;315(3): 245-246.

26. American Academy of Hospice and Palliative Medicine Advisory Brief: Guidance on Responding to Requests for Physician-Assisted Dying. 2016. http://aahpm.org/positions/padbrief. Accessed November 10, 2018.

27. Tulsky JA, Ciampa R, Rosen EJ; The University of Pennsylvania Center for Bioethics Assisted Suicide Consensus Panel. Responding to legal requests for aid-in-dying. Ann Intern Med 2000;132:494-499.

28. Ganzini L, Harvath TA, Jackson A, Goy ER, Miller LL, Delorit MA. Experiences of Oregon nurses and social workers with hospice patients who requested assistance with suicide. N Engl J Med 2002;347(8):582-589.

29. Miller LL, Harvath TA, Ganzini L, Goy ER, Delorit MA, Jackson A. Attitudes and experiences of Oregon hospice nurses and social workers regarding assisted suicide. Palliat Med 2004;18(8):685-691.

30. Faber-Langendoen K, Karlawish JH; The University of Pennsylvania Center for Bioethics Assisted Suicide Consensus Panel. Should assisted suicide be only physician assisted?. Ann Intern Med 2000;132:482-487.

31. Ganzini L, Nelson HD, Lee MA, Kraemer DF, Schmidt TA, Delorit MA. Oregon physicians' attitudes about and experiences with end-of-life care since Passage of the Oregon Death with Dignity Act. JAMA. 2001;285(18):2363-9. 